\title{
The risks and benefits of yoga for patients with chronic obstructive pulmonary disease: a systematic review and meta-analysis
}

Clinical Rehabilitation

2019, Vol. 33(I2) I847-1862

(C) The Author(s) 2019

Article reuse guidelines:

sagepub.com/journals-permissions DOI: $10.1177 / 026921551986055$ |

journals.sagepub.com/home/cre

@SAGE

\author{
Holger Cramer',2 (D, Heidemarie Haller', Petra Klose', \\ Lesley Ward ${ }^{2,3}$, Vincent $\mathrm{CH}$ Chung ${ }^{2,4}$ and Romy Lauche ${ }^{2,5}$
}

\begin{abstract}
Objectives: To determine the effectiveness and safety of yoga interventions on disease symptoms, quality of life and function in patients diagnosed with chronic obstructive pulmonary disease (COPD).

Data sources: Medline/PubMed, Scopus, and CENTRAL (Cochrane Central Register of Controlled Trials) were searched through 6 June 2019.

Review methods: Randomized controlled trials assessing the effects of yoga on quality of life, dyspnea, exercise capacity, and pulmonary function (FEVI) in patients with COPD were included. Safety was defined as secondary outcome. Mean differences (MD) and standardized mean differences (SMD) with 95\% confidence intervals (Cls) were computed. Risk of bias was assessed using the Cochrane tool.

Results: Eleven randomized controlled trials with a total of 586 patients were included. Meta-analysis revealed evidence for effects of yoga compared to no treatment on quality of life on the COPD Assessment Test ( $M D=3.8 \mathrm{I} ; 95 \% \mathrm{Cl}=0.97$ to $6.65 ; P=0.009, R^{2}=70 \%$ ), exercise capacity assessed by the 6 -minute walk test $\left(M D=25.53 \mathrm{~m} ; 95 \% \mathrm{Cl}=12.16 \mathrm{~m}\right.$ to $\left.38.90 \mathrm{~m} ; P=0.00 \mathrm{I}, R^{2}=0 \%\right)$, and pulmonary function assessed by $\mathrm{FEVI}$ predicted (MD $=3.95 \% ; 95 \% \mathrm{Cl}=2.74 \%$ to $5.17 \% ; P<0.00 \mathrm{I}, 12=0 \%$ ). Only the effects on exercise capacity and pulmonary function were robust against methodological bias. Effects were only present in breathing-focused yoga interventions but not in interventions including yoga postures. Adverse events were reported infrequently.

Conclusion: This meta-analysis found robust effects of yoga on exercise capacity and pulmonary function in patients with COPD. Yoga, specifically yoga breathing techniques, can be an effective adjunct intervention for patients with COPD. Yoga's safety needs to be assessed in more depth in future studies.
\end{abstract}

\footnotetext{
'Department of Internal and Integrative Medicine, Kliniken Essen-Mitte, Faculty of Medicine, University of DuisburgEssen, Essen, Germany

${ }^{2}$ Australian Research Centre in Complementary and Integrative Medicine (ARCCIM), Faculty of Health, University of Technology Sydney, Sydney, NSW, Australia ${ }^{3}$ Centre for Rehabilitation Research in Oxford (RRIO), Nuffield Department of Orthopaedics, Rheumatology \& Musculoskeletal Sciences (NDORMS), University of Oxford, Oxford, UK
}

\author{
${ }^{4}$ Jockey Club School of Public Health and Primary Care, \\ The Chinese University of Hong Kong, Hong Kong, China \\ ${ }^{5}$ Department of Internal and Integrative Medicine, \\ Sozialstiftung Bamberg, Bamberg, Germany \\ Corresponding author: \\ Holger Cramer, Department of Internal and Integrative Medicine, \\ Kliniken Essen-Mitte, Faculty of Medicine, University of Duisburg- \\ Essen, Am Deimelsberg 34a, 45276 Essen, Germany. \\ Email: h.cramer@kem-med.com
}




\section{Keywords}

Chronic obstructive pulmonary disease (COPD), yoga, breathing exercises, meta-analysis, systematic review, exercise

Received: 12 June 2018; accepted: 7 June 2019

\section{Introduction}

A 2015 Cochrane review found that rehabilitation programs, including physical exercise, were beneficial for improving health-related quality of life and exercise capacity in chronic obstructive pulmonary disease. ${ }^{1}$

The combination of physical, breathing, and meditative practices in traditional yoga may provide an alternative exercise activity for patients with chronic obstructive pulmonary disease. Interestingly, adults with chronic obstructive pulmonary disease are among those with the lowest utilization rate of yoga..$^{2,3}$ Conversely, adults with asthma are among the highest yoga users, ${ }^{2,3}$ with evidence suggesting yoga significantly improves quality of life and respiratory function in that clinical population. ${ }^{4}$ Chronic obstructive pulmonary disease has been a major focus of research identified in a bibliometric analysis of yoga research, ${ }^{5}$ with a large number of trials published in recent years. As the 2015 Cochrane review $^{1}$ specifically excluded yogic exercise interventions, the potential benefits of yoga have not been systematically assessed. As such, this current systematic review aims to determine the effectiveness and safety of yogic interventions in patients diagnosed with chronic obstructive pulmonary disease. We hypothesized that yoga would be more effective than no treatment in improving quality of life, dyspnea, exercise capacity, and pulmonary outcomes in patients diagnosed with chronic obstructive pulmonary disease.

\section{Methods}

We used the Preferred Reporting Items for Systematic Reviews and Meta-Analyses guidelines $^{6}$ and the recommendations of the Cochrane Collaboration $^{7}$ as a framework for the reporting structure of this review. The review protocol was not registered.

We included randomized controlled trials, randomized cross-over studies, and cluster-randomized trials on adult patients with chronic obstructive pulmonary disease, regardless of publication language. Studies on yoga interventions were included, regardless of yoga tradition, length or frequency of the yoga sessions, or intervention duration. Studies allowing individual co-interventions in addition to the study interventions were included. We grouped the yoga interventions according to their content:

1. Interventions including yoga breathing only (based on yoga theory and/or traditional yoga practices), without physical postures, meditation, or lifestyle advice.

2. Complex yoga interventions including yoga breathing and at least 1 of the following: physical postures, meditation, and/or lifestyle advice (based on yoga theory and/or practices).

Regarding control interventions, we included studies comparing yoga to no treatment, sham yoga, or any active control intervention.

In terms of outcome measures, studies were included if they assessed at least one of our primary outcomes:

1. Quality of life, assessed by validated diseasespecific or generic instruments. We preferred disease-specific instruments over generic ones when multiple instruments were used in a single study.

2. Dyspnea, assessed by validated self-report instruments.

3. Exercise capacity, clinician-assessed using standardized tests such as the 6-minute walk test. 
4. Pulmonary function, operationalized as absolute or predictive forced expiratory volume in one second, assessed by validated measures such as spirometry.

Safety of the intervention, assessed as the number of patients with adverse events, was defined as a secondary outcome.

On the basis of these eligibility criteria, we searched three electronic databases from their inception through 6 June 2019: Medline (via PubMed), Scopus, and the Cochrane Central Register of Controlled Trials (CENTRAL). We adapted the search strategy for each database as necessary. The complete search strategy for each database searched is given in the Supplemental Appendix. In addition, we manually screened reference lists of identified original articles or reviews, and the tables of contents of the International Journal of Yoga Therapy and the Journal of Yoga \& Physical Therapy.

Two reviewers independently conducted the abstract screening and selection process, and read in full potentially eligible articles. Disagreements over article inclusion were settled through discussion with a third reviewer until consensus was reached. If necessary, we obtained additional information from the authors of the primary study.

Two reviewers independently extracted data on demographics (e.g. age, gender, ethnicity), methods (e.g. randomization, allocation concealment), interventions (e.g. yoga type, frequency, duration), control interventions (e.g. type, frequency, duration), outcomes (e.g. outcome measures, assessment time points), and results using an a priori developed data extraction form. Likewise, two reviewers independently assessed risk of bias using the Cochrane risk of bias tool. This tool assesses risk of bias on seven criteria: random sequence generation, allocation concealment, blinding of participants and personnel, blinding of outcome assessment, incomplete outcome data, selective reporting, and other sources of bias. Each criteria is rated as either low, unclear, or high risk of bias. ${ }^{7}$ Discrepancies were checked with a third reviewer, and consensus was achieved by discussion.
In statistical analysis, we conducted separate meta-analyses for the different control conditions. We conducted meta-analyses using Review Manager software (Version 5.1; The Nordic Cochrane Centre, Copenhagen) by random effects model, using the generic inverse variance method if at least two studies assessing a specific outcome were available. We calculated standardized mean differences with $95 \%$ confidence intervals as the difference in means between groups divided by the pooled standard deviation. ${ }^{7}$ Where no standard deviations were available, we calculated them from standard errors, confidence intervals, or $t$-values, ${ }^{7}$ or contacted the trial authors by email for clarification. We defined a positive standardized mean difference as indicating beneficial effects of yoga compared to the control intervention (i.e. increased quality of life, exercise capacity, and pulmonary function), except for dyspnea where we defined a negative standardized mean difference as indicating beneficial effects (i.e. lower dyspnea). We used Cohen's ${ }^{8}$ categories to evaluate the magnitude of the overall effect size with standardized mean differences of (1) 0.2-0.5: small; (2) 0.5-0.8: medium, and (3) 0.8: large effect sizes. Where the same assessment instrument was used in multiple studies, we further calculated mean differences and their $95 \%$ confidence intervals as the absolute difference between mean values in the two groups. ${ }^{7}$ We further analyzed the magnitude of heterogeneity using the $R^{2}$ statistic. The statistic was categorized as (1) $I^{2}=0 \%-24 \%$ : low; (2) $I^{2}=25 \%-49 \%$ : moderate; (3) $I^{2}=50 \%-74 \%$ : substantial; and (4) $I^{2}=75 \%-100 \%$ : considerable heterogeneity. ${ }^{7} \mathrm{We}$ further used the chi-square test to assess whether differences in results were compatible with chance alone. Given the low power of this test when only few studies or studies with low sample size are included in a meta-analysis, we regarded a $P$-value equal or less than 0.10 to indicate significant heterogeneity. ${ }^{7}$

We conducted subgroup analyses for the type of yoga intervention (yoga breathing interventions vs complex yoga interventions). To test the robustness of significant results, we conducted sensitivity analyses for studies with high versus low risk of bias at the following domains: selection bias, detection 
bias, and attrition bias. If present in the respective meta-analysis, we also used subgroup and sensitivity analyses to explore possible reasons for statistical heterogeneity.

If at least 10 studies were included in a metaanalysis, we originally planned to generate funnel plots to assess publication bias. ${ }^{7}$ Since less than 10 studies were included in each meta-analysis, this was not possible.

\section{Results}

The results of the literature search are shown in Figure 1; Table 1 presents characteristics of the included studies, Table 2 the interventions used in the included studies, and Table 3 the risk of bias of the included studies. The 11 studies included in the review $^{9-21}$ had a combined total of 586 patients, with a median sample size of 49 patients. The median age of included patients was 57.6 years; the studies included a median of $16.7 \%$ female patients. The median duration of the yoga interventions was 12 weeks, with a median frequency of five yoga sessions per week.

Regarding patient-reported outcomes, our metaanalysis found no evidence for superiority of yoga to no treatment on quality of life or dyspnea when multiple outcome measures were included in the analysis (Figure 2). However, when we limited the analysis to studies using the chronic obstructive pulmonary disease assessment test, we found evidence for positive effects compared to no treatment (Table 4).

Regarding clinician-assessed outcomes and including multiple assessment methods in the analyses, we found positive effects of yoga compared to no treatment on exercise capacity, but not for forced expiratory volume in one second (Figure 3 ). When we limited the analysis to studies using the same assessment method, we found further effects of yoga compared to no treatment on predicted but not on absolute values of forced expiratory volume in one second (Table 4). We found no differences between yoga and physiotherapy on forced expiratory volume in one second.

In subgroup analyses, when limiting the analysis to studies using breathing-based yoga interventions, we found positive effects compared to no treatment on dyspnea, exercise capacity, and predicted forced expiratory volume in one second (Table 5). When limiting the analysis to complex yoga interventions, we only found effects on exercise capacity.

In sensitivity analyses, including only studies with low risk of selection bias, only the effects of yoga compared to no treatment on quality of life on the chronic obstructive pulmonary disease assessment test and on exercise capacity on the 6-minute walk test remained significant, based on a single study (72 patients). In studies with low risk of detection bias, only the effects on exercise capacity (6-minute walk test, 3 studies, 160 patients) and predicted forced expiratory volume in one second ( 2 studies, 88 patients) remained significant. Finally, when we considered only studies with low risk of attrition bias, the effects of yoga compared to no treatment on exercise capacity (5 studies, 228 patients) and predicted forced expiratory volume in one second ( 2 studies, 88 patients) remained significant.

Only 2 of the 11 studies reported safety-related outcomes. Of these, one study reported one serious adverse event (a case of cancer), but it was unclear in which group this event occurred. ${ }^{12}$ The other study reported $1(4 \%), 6(24 \%)$, and $4(16 \%)$ serious adverse events (hospitalizations; no reasons reported) in the yoga, no treatment, and physiotherapy groups, respectively. ${ }^{22}$ None of these studies reported whether the adverse events were intervention-related or not.

\section{Discussion}

In this systematic review and meta-analysis of 11 randomized trials on yoga for patients with chronic obstructive pulmonary disease, we found evidence for beneficial effects of yoga on disease-specific quality of life and physical outcomes. Both complex and breathing-based yoga interventions were effective for improving exercise capacity; breathing-based interventions additionally improved dyspnea and predicted forced expiratory volume. Only the effect on exercise capacity was robust against methodological bias. Reporting of safety was insufficient; however, where reported, rates of 


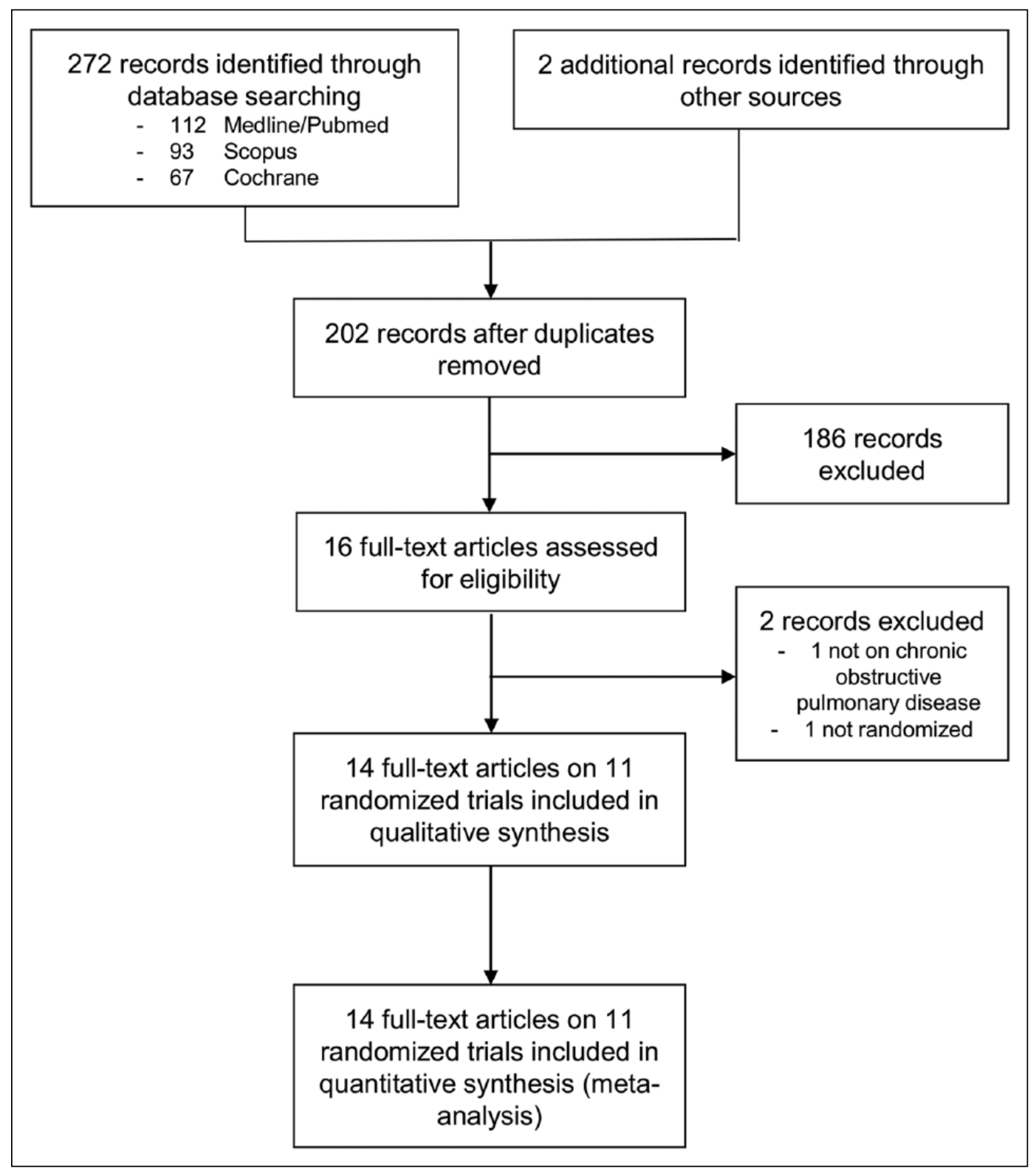

Figure I. Flowchart of the results of the literature search.

serious adverse events in the yoga group were lower than in both active (physiotherapy) and passive (no treatment) control groups.

Only one other systematic review on chronic obstructive pulmonary disease so far has exclusively targeted yoga interventions. ${ }^{23}$ The 2014 review, analyzing five studies, found evidence for positive effects of yoga on lung function and exercise capacity; however, patient-reported outcomes were not included, and the analysis did not differentiate 


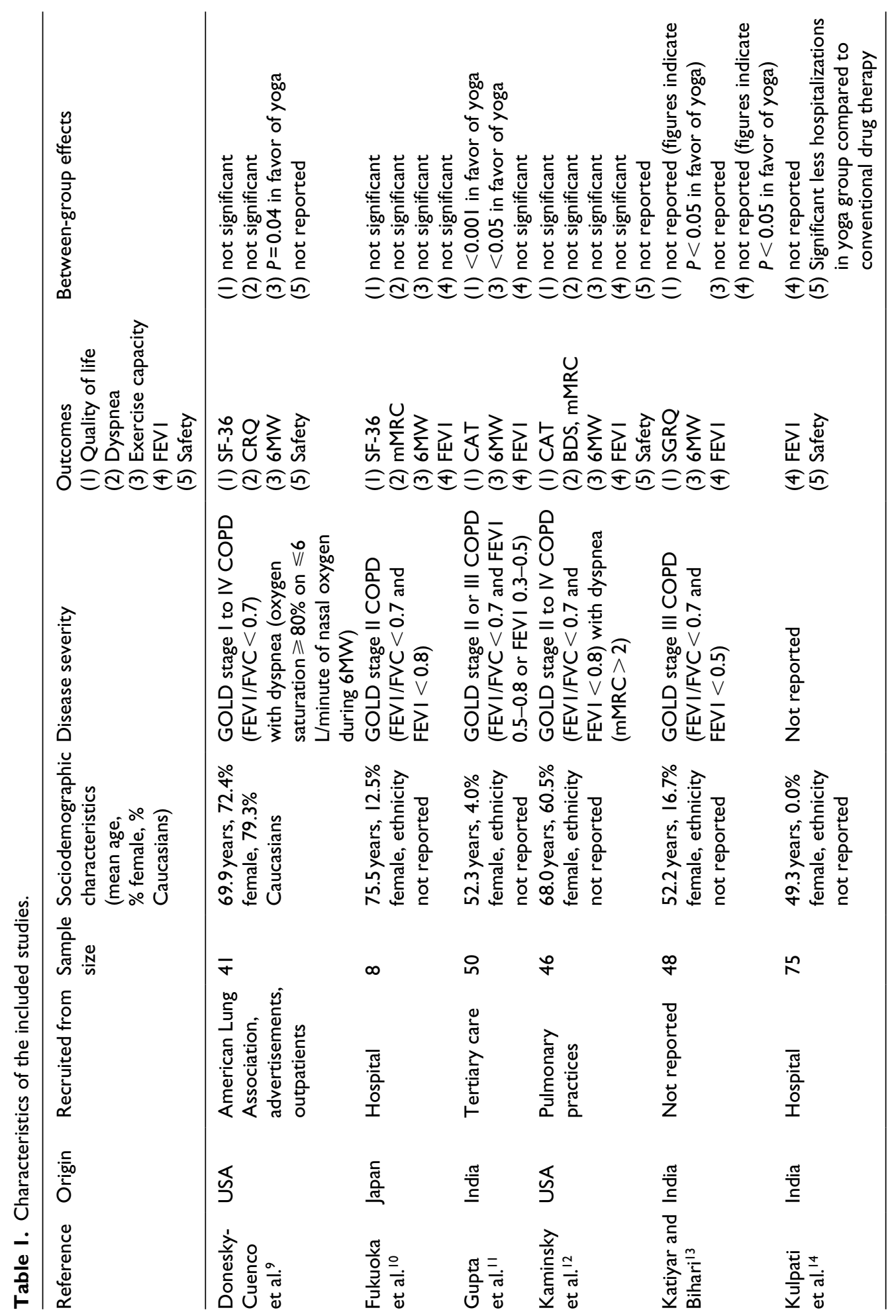




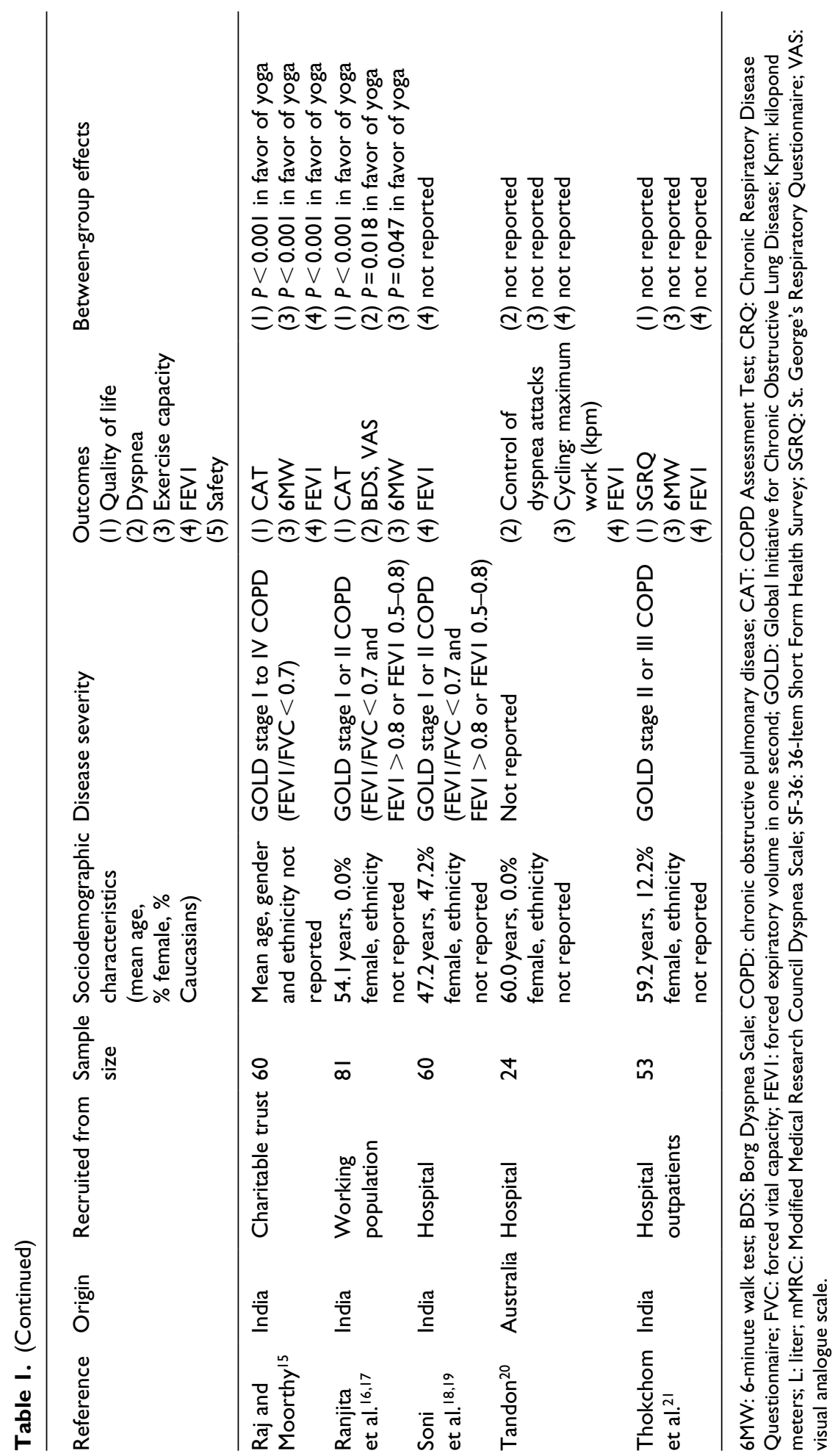




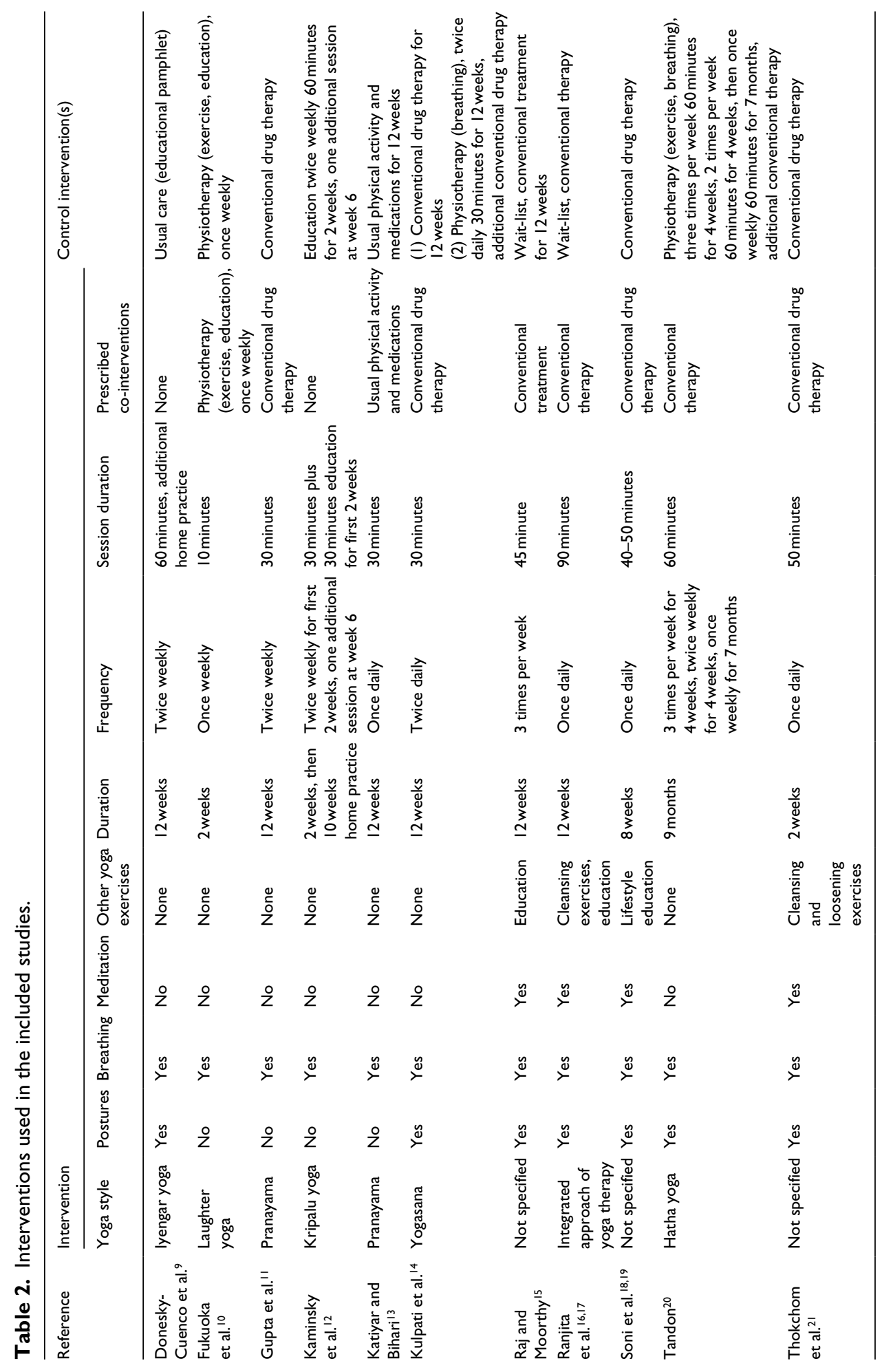




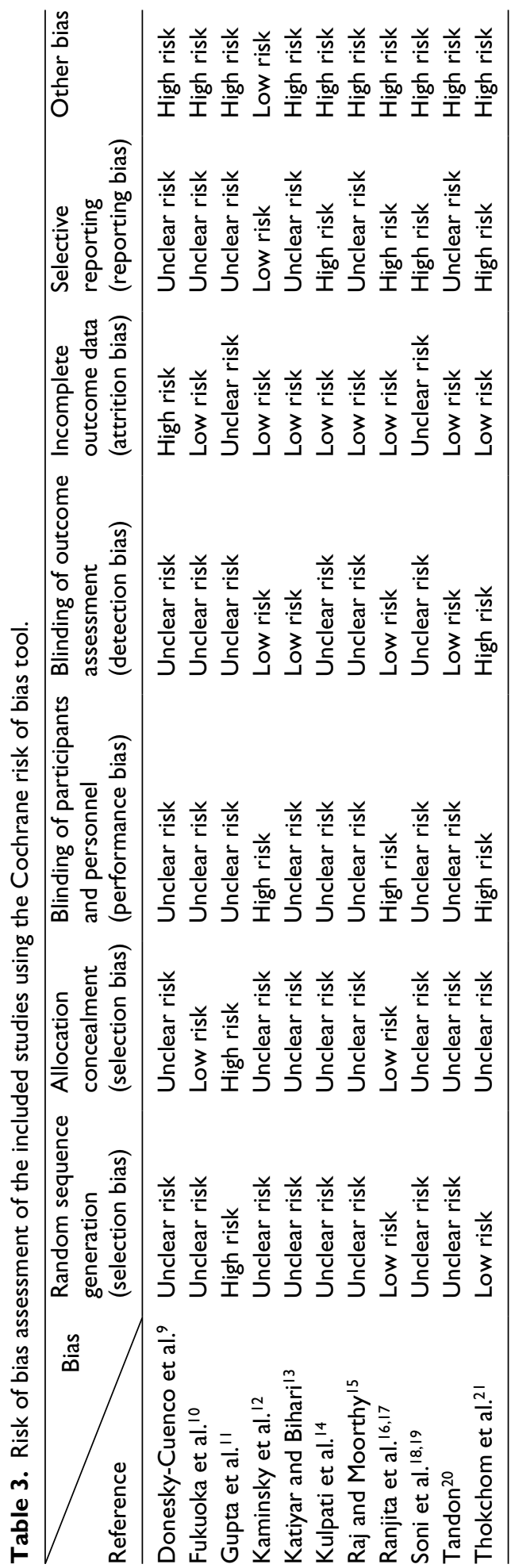




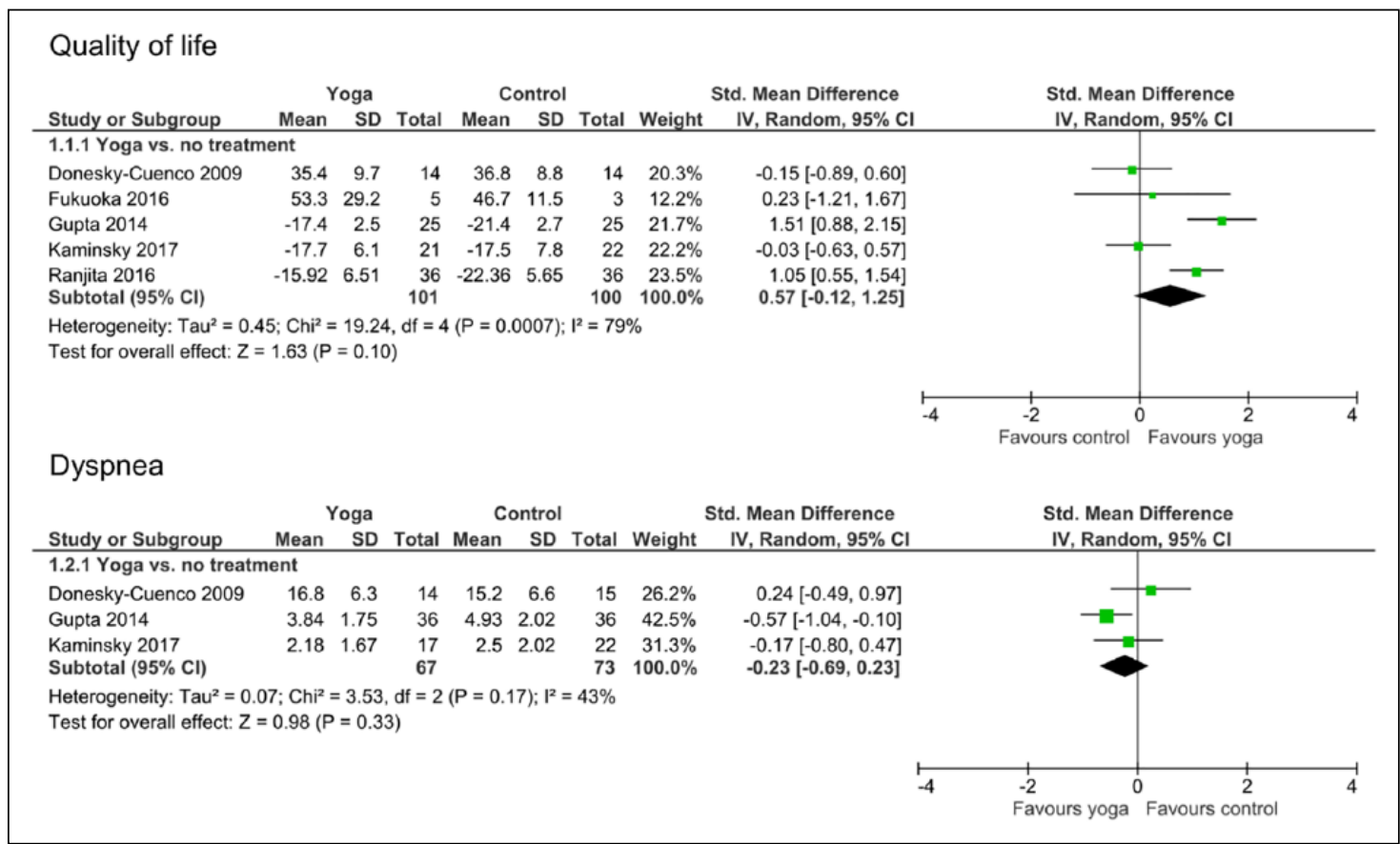

Figure 2. Effects of yoga compared to no treatment on patient-reported outcomes.

$\mathrm{Cl}$ : confidence interval; df: degrees of freedom; IV: inverse variance.

between different types of yoga. Due to the mostly unclear risk of bias of studies included in that review, and an increased number of studies on yoga interventions for chronic obstructive pulmonary disease published in subsequent years, an updated review seemed warranted.

The findings of this systematic review and meta-analysis are partly in line with those of earlier reviews on related interventions. In a Cochrane review, McCarthy et al. ${ }^{1}$ found exercise interventions beneficial for chronic obstructive pulmonary disease, but this review excluded yoga for not being aerobically demanding. A further Cochrane review $^{24}$ found limited evidence for breathing interventions; however, only 2 out of 16 included studies were on yoga-based breathing ${ }^{9}$ while the other studies used a variety of other types of breathing exercises.

There are several ways that yoga may benefit patients with chronic obstructive pulmonary disease. Physical activity is fundamental to the management of chronic obstructive pulmonary disease, ${ }^{1,25}$ and strongly correlated with functional exercise capacity. ${ }^{26}$ However, chronic obstructive pulmonary disease patients are significantly less active than sedentary healthy individuals, ${ }^{26}$ with physical activity being the strongest predictor of lower mortality in this patient group, an association partly explained by disease severity. ${ }^{26}$

Patients with chronic obstructive pulmonary disease are capable of physical activity at a level effective for physiological change, as manifested by a reduction in blood lactate and ventilation. ${ }^{27}$ While the practice of yoga commonly includes physical activity through the use of yoga postures, it has previously been questioned whether yoga postures are sufficiently physically demanding enough to reach these physiological levels of change in patients with chronic obstructive pulmonary disease. ${ }^{1}$ This review challenges this viewpoint, with results suggesting that physical yoga postures are associated with improved exercise capacity. In addition, yoga may play a broader role in the management of chronic obstructive 


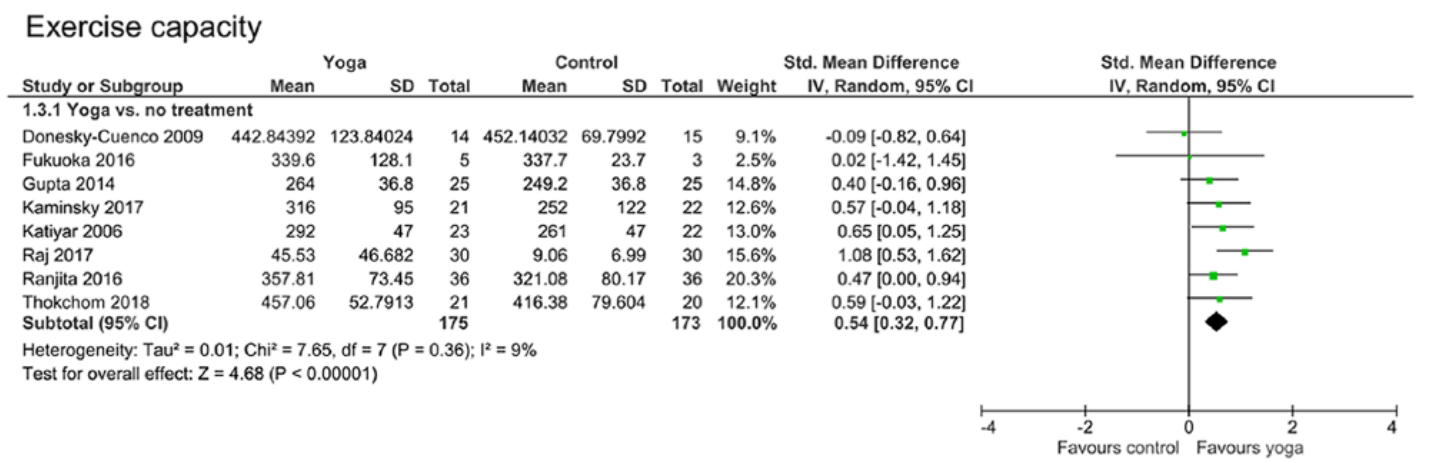

Forced expiratory volume in 1 second

\begin{tabular}{|c|c|c|c|c|c|c|c|c|c|c|c|}
\hline \multirow[b]{2}{*}{ Study or Subgroup } & \multicolumn{3}{|c|}{ Yoga } & \multicolumn{3}{|c|}{ Control } & \multicolumn{2}{|r|}{ Std. Mean Difference } & \multirow{2}{*}{\multicolumn{3}{|c|}{$\begin{array}{l}\text { Std. Mean Difference } \\
\text { IV, Random, } 95 \% \mathrm{Cl}\end{array}$}} \\
\hline & Mean & SD & Total & Mean & SD & Total & Weight & IV, Random, $95 \% \mathrm{CI}$ & & & \\
\hline \multicolumn{12}{|c|}{ 1.4.1 Yoga vs. no treatment } \\
\hline Donesky-Cuenco 2009 & 51.2 & 10.6 & 14 & 45.9 & 20.2 & 15 & $11.2 \%$ & $0.32[-0.42,1.05]$ & & & \\
\hline Fukuoka 2016 & 0.88 & 0.22 & 5 & 1.69 & 0.52 & 3 & $4.7 \%$ & $-2.01[-4.00,-0.02]$ & & & \\
\hline Gupta 2014 & 53.3 & 8.8 & 25 & 49.1 & 8.7 & 25 & $12.2 \%$ & $0.47[-0.09,1.04]$ & & & \\
\hline Kaminsky 2017 & 45 & 14 & 21 & 43 & 13 & 22 & $12.0 \%$ & $0.15[-0.45,0.74]$ & & & \\
\hline Katiyar 2006 & 52 & 2.1 & 23 & 48 & 2.1 & 22 & $11.3 \%$ & $1.87[1.16,2.58]$ & & & \\
\hline Kulpati 1982 & 1.112 & 0.3943 & 25 & 1.348 & 0.4434 & 25 & $12.2 \%$ & $-0.55[-1.12,0.01]$ & & & \\
\hline Raj 2017 & 0.078 & 0.444 & 30 & 0.024 & 0.023 & 30 & $12.6 \%$ & $0.17[-0.34,0.68]$ & & & \\
\hline Soni 2012 & 1.82 & 0.49 & 30 & 1.42 & 0.23 & 30 & $12.4 \%$ & $1.03[0.49,1.57]$ & & 1 & \\
\hline $\begin{array}{l}\text { Thokchom } 2018 \\
\text { Subtotal }(95 \% \mathrm{Cl})\end{array}$ & 1.9 & 0.4583 & $\begin{array}{r}21 \\
194\end{array}$ & 1.31 & 0.3578 & $\begin{array}{r}20 \\
192\end{array}$ & $\begin{array}{r}11.4 \% \\
100.0 \%\end{array}$ & $\begin{array}{r}1.40[0.71,2.09] \\
0.47[-0.06,1.00]\end{array}$ & & & \\
\hline \multicolumn{12}{|c|}{$\begin{array}{l}\text { Heterogeneity: } \text { Tau }^{2}=0.52 ; \mathrm{Ch}^{2}=47.23 \text {, df }=8(P<0.00001) ; I^{2}=83 \% \\
\text { Test for overall effect: } Z=1.72(P=0.08)\end{array}$} \\
\hline \multicolumn{12}{|c|}{ 1.4.2 Yoga vs. physiotherapy } \\
\hline Kulpati 1982 & 1.112 & 0.3943 & 25 & 1.137 & 0.5614 & 25 & $64.1 \%$ & $-0.05[-0.61,0.50]$ & & & \\
\hline $\begin{array}{l}\text { Tandon } 1978 \\
\text { Subtotal }(95 \% \mathrm{Cl})\end{array}$ & 0.022 & 0.212 & $\begin{array}{l}12 \\
37\end{array}$ & -0.0815 & 0.1663 & $\begin{array}{l}12 \\
37\end{array}$ & $\begin{array}{r}35.9 \% \\
100.0 \%\end{array}$ & $\begin{array}{l}0.52[-0.29,1.34] \\
0.16[-0.39,0.70]\end{array}$ & & & \\
\hline \multicolumn{12}{|c|}{$\begin{array}{l}\text { Heterogeneity: } \text { Tau }^{2}=0.04 ; \mathrm{Chi}^{2}=1.31, \mathrm{df}=1(P=0.25) ; 1^{2}=23 \% \\
\text { Test for overall effect: } Z=0.56(P=0.57)\end{array}$} \\
\hline & & & & & & & & & $\begin{array}{cc}-2 & 0 \\
\text { Favours control } & 0\end{array}$ & $\begin{array}{r}2 \\
\text { Favours yoga }\end{array}$ & 4 \\
\hline
\end{tabular}

Figure 3. Effects of yoga compared to no treatment and physiotherapy on clinician-assessed outcomes. $\mathrm{Cl}$ : confidence interval; df: degrees of freedom; IV: inverse variance.

pulmonary disease, through the positive impact of breathing-based yoga styles on parameters of dyspnea and predicted forced expiratory volume. Procedurally, breathing-based yoga and inspiratory muscle training share some similarity. In a recent systematic review, inspiratory muscle training is also found to be effective in decreasing dyspnea, ${ }^{28}$ which may suggest their common mechanism of action. A small randomized trial conducted among institutionalized frail older adults showed that breathing-based yoga is superior to inspiratory muscle training in respiratory strength and endurance, ${ }^{29}$ but their comparative effectiveness among patients with chronic obstructive pulmonary disease on clinical relevant outcomes are yet to be evaluated. 


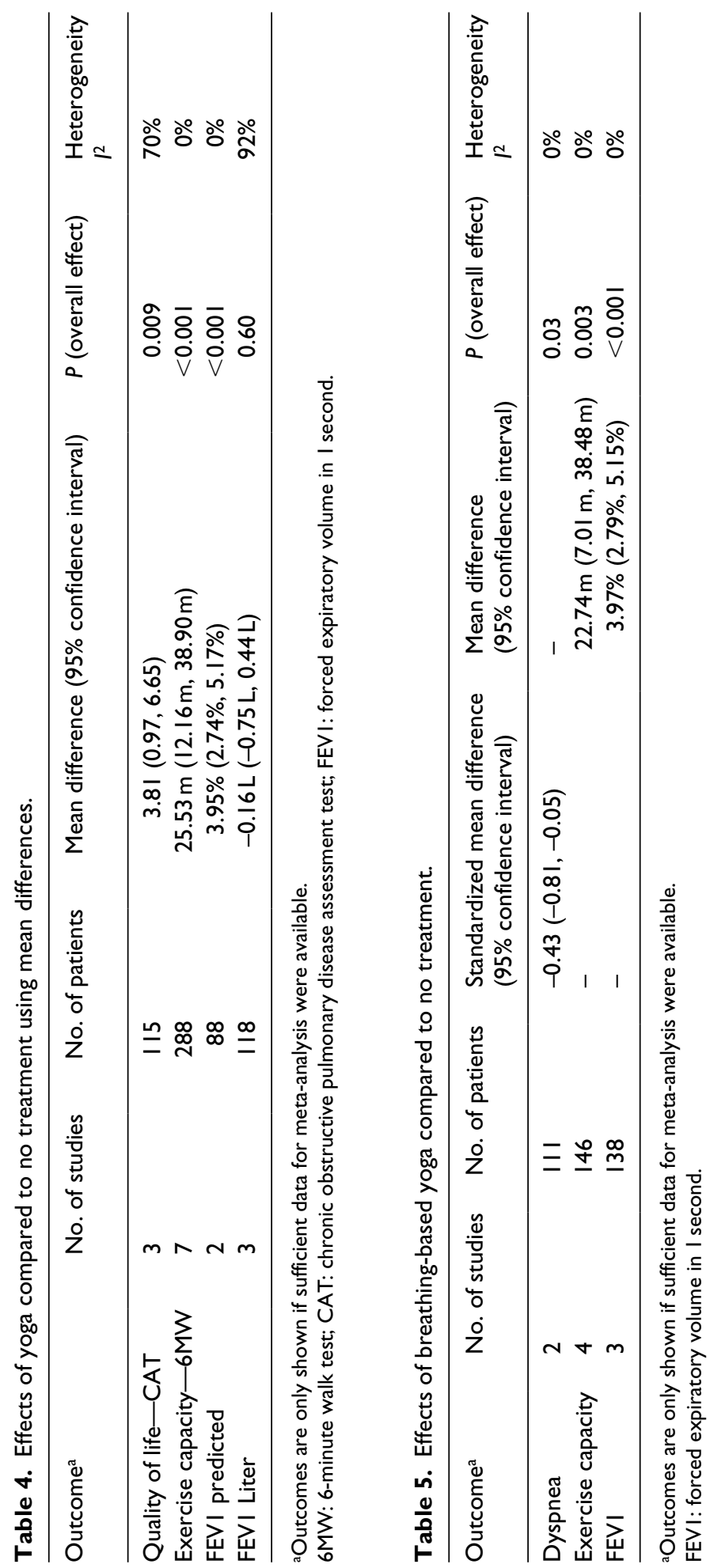


Comparable effects of yoga are indicated in patients with hypertension, and were attributed to the effects of yoga breathing on sympathetic activation. ${ }^{30,31}$ Increasing parasympathic activity and decreasing sympathetic activation is regarded as a primary mechanism of yoga therapy. ${ }^{30,32}$ Specifically, yoga breathing and relaxation exercises have been shown to increase parasympathetic activity both in healthy individuals $\mathrm{s}^{33,34}$ and in patients with chronic obstructive pulmonary disease. ${ }^{35,36}$ Importantly, the addition of yoga postures does not further increase this effect, ${ }^{33}$ meaning patients with reduced physical capacity may gain benefit from yoga through the practice of yogabased breathing techniques alone. Our results also support the role of breathing in chronic conditions with similar sympathetic activation, such as cardiovascular conditions. ${ }^{36}$ Slow breathing, integral to many yoga breathing exercises, has been shown to positively influence dyspnea and improve resting pulmonary gas exchange and exercise performance in patients with chronic heart failure, ${ }^{37}$ possibly by reducing chemoreflex sensitivity. ${ }^{38}$

A strength of this review is its adherence with Preferred Reporting Items for Systematic Reviews and Meta-Analyses guidelines, the comprehensive literature search, and the rigorous predefined methods of analysis. The latter is important if yoga is to be used as an adjunct intervention for patients with chronic obstructive pulmonary disease. Although twice as many studies were available for this metaanalysis compared to the first one, the relatively small number of studies and their low sample and varying quality sizes still are the main limitations of this analysis. The interventions differed between studies making comparisons difficult, especially for adverse events. Safety was infrequently reported. We were thus unable to draw definite conclusions on the intervention's safety. Moreover, it is possible that this review missed some studies not listed in the standard databases.

The results of this review are further limited by the validity of the available data, and the paucity of safety reporting in trials to date. While attrition bias was generally low across all studies, a lack of transparency in treatment selection, data collection, and reporting of analysis limits the internal robustness of the trials. Many of these issues concern the reporting, rather than the content, of trials. As such, the clinical use of yoga for the management of chronic obstructive pulmonary disease would be greatly enhanced by improved reporting of clinical yoga trials in line with accepted guidelines such as the Consolidated Standards of Reporting Trials, ${ }^{39}$ improving the methodological transparency of trials. The additional use of reporting guidelines for intervention content $t^{40}$ and safety ${ }^{41}$ in future research will aid the implementation of research into practice, by providing clinicians and yoga therapists with clear detail on the type and amount of yoga considered effective in chronic obstructive pulmonary disease management.

The participant demographics in the included studies are reflective of chronic obstructive pulmonary disease patients in the general population, making the results generalizable to clinical practice. Chronic obstructive pulmonary disease patients are typically first diagnosed in their 40s, with incidence slightly higher in men, and increasing with age. ${ }^{38} \mathrm{~A}$ multidisciplinary program of pulmonary rehabilitation is recommended for all chronic obstructive pulmonary disease patients. ${ }^{42}$ Current programs tend to be physically based, with content focused on aerobic and strength-based exercise, such as walking, cycling, and resistance training. ${ }^{43}$ Our review suggests the addition of breathing-based exercises may enhance these programs, through the improvement of exercise capacity. Furthermore, current guidelines recognize the likely presence of anxiety and depression in individuals with this chronic health problem, and recommend the integration of psychosocial management into rehabilitation program. ${ }^{44}$ This review suggests yoga may positively affect quality of life in chronic obstructive pulmonary disease patients. In addition, prior reviews indicate yoga as a safe and effective option for the management of anxiety and depression. ${ }^{45,46}$

Importantly, the low attrition rates across the included studies in this review suggest yoga as an acceptable form of physical and psychosocial symptom management for individuals with chronic obstructive pulmonary disease. This acceptance reflects trends in the general population, with the increased popularity of yoga for the management 
of chronic and mental health conditions. ${ }^{2,47}$ With physical and breathing exercises commonly included in both instructor-delivered and selfmanaged yoga practice ${ }^{48,49}$ yoga presents a viable option for the symptom management of chronic obstructive pulmonary disease. Future research into the content and dosage of effective yoga practice will further aid clinical recommendations for practice. More high-quality trials with adequate sample size and low risk of bias are needed.

\section{Clinical messages}

- Yoga may be an effective option for improving physical capacity and breathing parameters in chronic obstructive pulmonary disease rehabilitation.

- The safety of yoga in chronic obstructive pulmonary disease is inconclusive due to insufficient safety reporting in clinical trials to date.

\section{Author contributions}

H.C. and R.L. were responsible for conception and design. H.C., H.H., and P.K. were responsible for the analysis of the data. All authors made substantial contributions to conception and design and interpretation of the data, drafted the manuscript, gave final approval of the version to be published, and agreed to be accountable for all aspects of the work in ensuring that questions related to the accuracy or integrity of any part of the work are appropriately investigated and resolved.

\section{Declaration of conflicting interests}

The author(s) declared no potential conflicts of interest with respect to the research, authorship, and/or publication of this article.

\section{Funding}

The author(s) disclosed receipt of the following financial support for the research, authorship and/or publication of this article: $\mathrm{HC}$ was supported by the Erich Rothenfußer Stiftung.

\section{Supplemental material}

Supplemental material for this article is available online.

\section{ORCID iD}

Holger Cramer iD https://orcid.org/0000-0002-3640-8046

\section{References}

1. McCarthy B, Casey D, Devane D, et al. Pulmonary rehabilitation for chronic obstructive pulmonary disease. Cochrane Database Syst Rev 2015; (2): CD003793, https://www.ncbi.nlm.nih.gov/pubmed/25705944

2. Birdee GS, Legedza AT, Saper RB, et al. Characteristics of yoga users: results of a national survey. J Gen Intern Med 2008; 23(10): 1653-1658.

3. Birdee GS, Yeh GY, Wayne PM, et al. Clinical applications of yoga for the pediatric population: a systematic review. Acad Pediatr 2009; 9(4): 212.e1-9-220.e1-9.

4. Cramer H, Posadzki P, Dobos G, et al. Yoga for asthma: a systematic review and meta-analysis. Ann Allergy Asthma Immunol 2014; 112(6): 503.e5-510.e5.

5. Cramer H, Lauche R and Dobos G. Characteristics of randomized controlled trials of yoga: a bibliometric analysis. BMC Complement Altern Med 2014; 14: 328.

6. Moher D, Liberati A, Tetzlaff J, et al. Preferred reporting items for systematic reviews and meta-analyses: the PRISMA statement. BMJ 2009; 6: e1000097.

7. Higgins JPT and Green S. Cochrane handbook for systematic reviews of interventions. West Sussex: John Wiley \& Sons, Ltd., 2008.

8. Cohen J. Statistical power analysis for the behavioral sciences. Hillsdale, NJ: Lawrence Erlbaum Associates, 1998.

9. Donesky-Cuenco D, Nguyen HQ, Paul S, et al. Yoga therapy decreases dyspnea-related distress and improves functional performance in people with chronic obstructive pulmonary disease: a pilot study. J Altern Complement Med 2009; 15(3): 225-234.

10. Fukuoka A, Ueda M, Ariyama Y, et al. Effect of laughter yoga on pulmonary rehabilitation in patients with chronic obstructive pulmonary disease. J Nara Med Assoc 2016; 67(1-3): 11-20.

11. Gupta A, Gupta R, Sood S, et al. Pranayam for treatment of chronic obstructive pulmonary disease: results from a randomized, controlled trial. Integr Med 2014; 13(1): 26-31.

12. Kaminsky DA, Guntupalli KK, Lippmann J, et al. Effect of yoga breathing (pranayama) on exercise tolerance in patients with chronic obstructive pulmonary disease: a randomized, controlled trial. J Altern Complement Med 2017; 23(9): 696-704.

13. Katiyar SK and Bihari S. Role of pranayama in the rehabilitation of COPD patients - a randomized controlled study. Ind J Allerg Appl Immunol 2006; 20(2): 98-104.

14. Kulpati DD, Kamath RK and Chauhan MR. The influence of physical conditioning by yogasanas and breathing exercises in patients of chronic obstructive lung disease. $J$ Assoc Physicians India 1982; 30(12): 865-868.

15. Raj N and Moorthy AM. Yoga-based pulmonary rehabilitation for the management of dyspnea in coal miners with 
chronic obstructive pulmonary disease: a randomized controlled trial. Int J Res Sci Technol 2017; 7(2): 113-128.

16. Ranjita R, Badhai S, Hankey A, et al. A randomized controlled study on assessment of health status, depression, and anxiety in coal miners with chronic obstructive pulmonary disease following yoga training. Int J Yoga 2016; 9(2): 137-144.

17. Ranjita R, Hankey A, Nagendra HR, et al. Yoga-based pulmonary rehabilitation for the management of dyspnea in coal miners with chronic obstructive pulmonary disease: a randomized controlled trial. $J$ Ayurveda Integr Med 2016; 7(3): 158-166.

18. Soni R, Munish K, Singh K, et al. Study of the effect of yoga training on diffusion capacity in chronic obstructive pulmonary disease patients: a controlled trial. Int J Yoga 2012; 5(2): 123-127.

19. Soni R, Singh S and Singh KP. The effect of pranayama and yogasana on lung functions in mild and moderate chronic obstructive pulmonary disease. Biomedicine 2012; 32(1): 57-60.

20. Tandon MK. Adjunct treatment with yoga in chronic severe airways obstruction. Thorax 1978; 33(4): 514-517.

21. Thokchom SK, Gulati K, Ray A, et al. Effects of yogic intervention on pulmonary functions and health status in patients of COPD and the possible mechanisms. Complement Ther Clin Pract 2018; 33: 20-26.

22. Kulpati DDS and Kamath RK. The influence of physical conditioning by yogasanas and breathing exercises in patients of chronic obstructive pulmonary disease. Lung India 1982; 1(1): 35-38.

23. Liu XC, Pan L, Hu Q, et al. Effects of yoga training in patients with chronic obstructive pulmonary disease: a systematic review and meta-analysis. $J$ Thorac Dis 2014; 6(6): 795-802.

24. Holland AE, Hill CJ, Jones AY, et al. Breathing exercises for chronic obstructive pulmonary disease. Cochrane Database Syst Rev 2012; 10: CD008250.

25. Global Initiative for Chronic Obstructive Lung Disease. From the global strategy for the diagnosis, management and prevention of COPD, global initiative for chronic obstructive lung disease (GOLD), 2017, http://goldcopd .org (2017, accessed 30 April 2018).

26. Waschki B, Kirsten A, Holz O, et al. Physical activity is the strongest predictor of all-cause mortality in patients with COPD: a prospective cohort study. Chest 2011; 140(2): 331-342.

27. Casaburi R, Patessio A, Ioli F, et al. Reductions in exercise lactic acidosis and ventilation as a result of exercise training in patients with obstructive lung disease. $\mathrm{Am} \mathrm{Rev}$ Respir Dis 1991; 143(1): 9-18.

28. Beaumont M, Forget P, Couturaud F, et al. Effects of inspiratory muscle training in COPD patients: a systematic review and meta-analysis. Clin Respir J 2018; 12(7): 2178-2188.

29. Cebria i, Iranzo M, Arnall DA, Igual Camacho C, et al. Effects of inspiratory muscle training and yoga breathing exercises on respiratory muscle function in institutionalized frail older adults: a randomized controlled trial. $J$ Geriatr Phys Ther 2014; 37(2): 65-75.

30. Cramer H. The efficacy and safety of yoga in managing hypertension. Exp Clin Endocrinol Diabetes 2016; 124(2): 65-70.

31. Cramer H, Haller H, Lauche R, et al. A systematic review and meta-analysis of yoga for hypertension. Am J Hypertens 2014; 27(9): 1146-1151.

32. Stair DC, Rios WA and Black HR. Atypical causes of curable renovascular hypertension: a review. Prog Cardiovasc Dis 1990; 33(3): 185-210.

33. Markil N, Whitehurst M, Jacobs PL, et al. Yoga Nidra relaxation increases heart rate variability and is unaffected by a prior bout of Hatha yoga. J Altern Complement Med 2012; 18(10): 953-958.

34. Mourya M, Mahajan AS, Singh NP, et al. Effect of slowand fast-breathing exercises on autonomic functions in patients with essential hypertension. $J$ Altern Complement Med 2009; 15(7): 711-717.

35. Pomidori L, Campigotto F, Amatya TM, et al. Efficacy and tolerability of yoga breathing in patients with chronic obstructive pulmonary disease: a pilot study. J Cardiopulm Rehabil Prev 2009; 29(2): 133-137.

36. Bernardi L, Porta C, Spicuzza L, et al. Slow breathing increases arterial baroreflex sensitivity in patients with chronic heart failure. Circulation 2002; 105(2): 143-145.

37. Bernardi L, Spadacini G, Bellwon J, et al. Effect of breathing rate on oxygen saturation and exercise performance in chronic heart failure. Lancet 1998; 351(9112): 1308-1311.

38. Spicuzza L, Gabutti A, Porta C, et al. Yoga and chemoreflex response to hypoxia and hypercapnia. Lancet 2000; 356(9240): 1495-1496.

39. Boutron I, Moher D, Altman DG, et al. Extending the CONSORT statement to randomized trials of nonpharmacologic treatment: explanation and elaboration. Ann Intern Med 2008; 148(4): 295-309.

40. Slade SC, Dionne CE, Underwood M, et al. Consensus on exercise reporting template (CERT): modified Delphi study. Phys Ther 2016; 96(10): 1514-1524.

41. Ioannidis JP, Evans SJ, Gotzsche PC, et al. Better reporting of harms in randomized trials: an extension of the CONSORT statement. Ann Intern Med 2004; 141(10): 781-788.

42. The National Institute for Health and Care Excellence. Chronic obstructive pulmonary disease in over 16s: diagnosis and management, https://www.nice.org.uk/guidance /cg101 (2010, accessed 14 April 2018).

43. The National Institute for Health and Care Excellence. Chronic obstructive pulmonary disease: management of chronic obstructive pulmonary disease in adults in primary and secondary care [CG101], https://www.nice .org.uk/guidance/CG101/uptake (2010, accessed 14 April 2018).

44. The National Institute for Health and Care Excellence. Depression in adults with a chronic physical health 
problem:recognitionandmanagement,https://www.nice.org. uk/guidance/CG91/chapter/1-Guidance\#step-1-recognition -assessment-and-initial-management-in-primary-care-and -general-hospital (2009, accessed 18 April 2018).

45. Cramer H, Lauche R, Langhorst J, et al. Yoga for depression: a systematic review and meta-analysis. Depress Anxiety 2013; 30(11): 1068-1083.

46. Cramer H, Lauche R, Anheyer D, et al. Yoga for anxiety: a systematic review and meta-analysis of randomized controlled trials. Depress Anxiety 2018; 35(9): 830-843.
47. Saper RB, Eisenberg DM, Davis RB, et al. Prevalence and patterns of adult yoga use in the United States: results of a national survey. Altern Ther Health Med 2004; 10(2): 44-49.

48. Cramer H, Ward L, Steel A, et al. Prevalence, patterns, and predictors of yoga use: results of a U.S. nationally representative survey. Am J Prev Med 2016; 50(2): 230-235.

49. Penman S, Cohen M, Stevens P, et al. Yoga in Australia: results of a national survey. Int J Yoga 2012; 5(2): 92-101. 


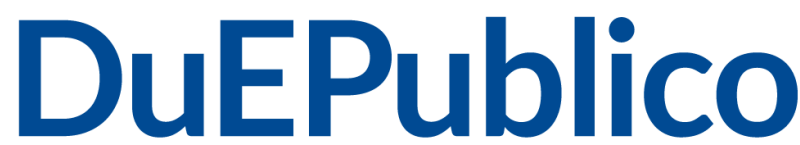

Duisburg-Essen Publications online
UNIVERSITÄT

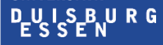

Offen im Denken

Ub $\mid \begin{aligned} & \text { universitäts } \\ & \text { bibliothek }\end{aligned}$

This text is made available via DuEPublico, the institutional repository of the University of Duisburg-Essen. This version may eventually differ from another version distributed by a commercial publisher.

DOI: $\quad 10.1177 / 0269215519860551$

URN: urn:nbn:de:hbz:464-20210505-160801-4

This publication is with permission of the rights owner freely accessible due to an Alliance licence and a national licence (funded by the DFG, German Research Foundation) respectively.

(C) The Author(s) 2019. All rights reserved. 